\title{
FFHD
}

Functional Foods in Health and Disease

\section{Hypoglycemic effects of antioxidant-rich plant extracts on postprandial glycemic responses in participants with prediabetes (GLARE study)}

\author{
Wen Xin Janice Lim ${ }^{1}$, Cheryl S. Gammon ${ }^{1}$, Pamela R. von Hurst ${ }^{2}$, Lynne Chepulis ${ }^{3}$, Owen Mugridge ${ }^{2}$, \\ Rachel A. Page P,5* $^{*}$
}

\begin{abstract}
${ }^{1}$ School of Health Sciences, Massey University, Auckland 0632, New Zealand; ${ }^{2}$ School of Sport, Exercise and Nutrition, Massey University, Auckland 0632, New Zealand; ${ }^{3}$ Waikato Medical Research Centre, Te Huataki Waiora School of Health, University of Waikato, Hamilton 3216, New Zealand; ${ }^{4}$ School of Health Sciences, Massey University, Wellington 6021, New Zealand; ${ }^{5}$ Centre for Metabolic Health Research, Massey University, Auckland 0632, New Zealand
\end{abstract}

*Corresponding Author: Rachel A. Page, PhD, Associate Professor, School of Health Sciences, Massey University, Wellington 6021, New Zealand and Centre for Metabolic Health Research, Massey University, Auckland 0632, New Zealand

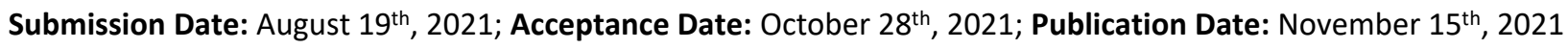

Please cite this article as: Lim W.X.J., Gammon C.S., von Hurst P.R., Chepulis L., Mugridge O., Page R.A. Hypoglycaemic effects of antioxidant-rich plant extracts on postprandial glycaemic responses in participants with prediabetes (GLARE study). Functional Foods in Health and Disease 2021; 11(11): 604-626. DOI: https://www.doi.org/10.31989/ffhd.v11i11.829

\section{ABSTRACT}

Background: Plant extracts may help to improve glycemic control in individuals with poor glycemic control. However, few studies have been investigated in the prediabetes cohort, which is a high-risk condition for T2DM. Thus, this study aimed to investigate the acute effect of grape seed, rooibos tea, and olive leaf extracts on postprandial blood glucose and insulin in participants with prediabetes.

Methods: An acute, single-blind, placebo-controlled, non-randomized, crossover study (ACTRN12617000837325) where placebo and extracts of grape seed, rooibos tea and olive leaf standardized for total antioxidant capacity were given separately during an oral glucose tolerance test to participants ( $n=19$, five men and fourteen women, aged 65.0 \pm 1.6 years, Body Mass Index (BMI) $27.3 \pm 1.1 \mathrm{~kg} / \mathrm{m}^{2}$ ) with prediabetes (Glycated hemoglobin A1c (HbA1c) $42 \pm 1$ $\mathrm{mmol} / \mathrm{mol}$ ). The primary outcome incremental area under the curve of glucose ( $\mathrm{i} A \cup \mathrm{C}_{\mathrm{glucose}}$ ) was examined with other glycemic measures. Data was analyzed using linear mixed model for repeated measures. Secondary analysis was conducted by stratifying participants into either a healthier or less healthy subgroup based on the postprandial time to glucose and insulin peaks, with the less healthy subgroup experiencing delayed glucose and/or insulin peaks. 
Results: There were no overall significant changes to glucose and insulin measures between all plant extracts and placebo $(p>0.05)$. Upon secondary analysis, all extracts affected glycemic responses in the less healthy subgroup. Compared to placebo, grape seed reduced plasma iAUC $C_{\text {glucose }}(p=0.016,21.9 \%$ reduction), $2 \mathrm{~h}$ postprandial glucose ( $2 \mathrm{hPG})(p=0.034,14.7 \%$ reduction) and metabolic clearance rate of glucose (MCR glucose) $(p=0.016,16.7 \%$ increase). It also improved insulin indices such as $2 \mathrm{~h}$ postprandial insulin $(2 \mathrm{hPI})(p=0.029,22.4 \%$ reduction) and Stumvoll overall insulin sensitivity index (ISI overall) $(p=0.028,15.0 \%$ increase). Rooibos tea extract significantly improved $\beta$-cell function as demonstrated by the increased oral disposition index (DI) ( $p=0.031,32.4 \%$ increase) compared to placebo. Olive leaf extract significantly increased incremental area under the curve of insulin ( iAUC $\left._{\text {insulin }}\right)(p=0.040,16.7 \%$ increase).

Conclusion: Grape seed, rooibos tea and olive leaf extracts demonstrated acute hypoglycemic benefits in adults with prediabetes and having less healthy metabolic profiles. A chronic study on the plant extracts is warranted to determine their longer-term impact on prediabetes.

\section{Trial Registration ID: ACTRN12617000837325}

Keywords: grape seed extract; rooibos tea extract; olive leaf extract; impaired glycemic control; hyperglycemia; hypoglycemic effects; insulin sensitivity; beta-cell function

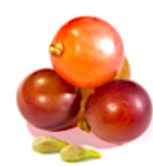

Grape seed extract

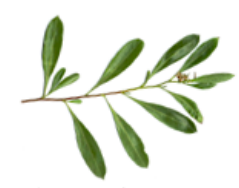

Olive leaf extract

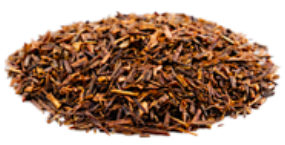

Rooibos tea extract

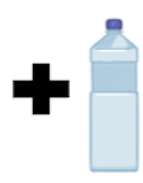

Oral glucose tolerance test (OGTT)

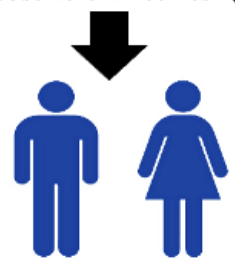

Impact on glycaemic responses in less healthy $(n=9)$ prediabetes subgroups:

- Grape seed extract reduced incremental area under the curve of glucose (iAUC glucose $(p=0.016)$ and $2 \mathrm{~h}$ postprandial glucose $(2 \mathrm{hPG})(p=0.034)$, and improved overall insulin sensitivity ( ISI $\left._{\text {overall }}\right)(p=0.028)$

- Rooibos tea extract improved $\beta$-cell function oral disposition index (DI) $(p=0.031)$

- Olive leaf extract increased incremental area under the curve of insulin (iAUC insulin $_{\text {) }}(p=0.040)$

(CFFC 2021. This is an Open Access article distributed under the terms of the Creative Commons Attribution 4.0 License (http://creativecommons.org/licenses/by/4.0) 


\section{INTODUCTION}

Prediabetes is characterized by isolated impaired fasting glucose (IFG) or isolated impaired glucose tolerance (IGT), or a combination of both (IFG/IGT) [1]. It is the intermediate state of hyperglycemia with blood glucose levels above normal but below the threshold to be classified as type 2 diabetes mellitus (T2DM) [2]. It is estimated that globally, 374 million (ages 20-79 years) (7.5\%) people have prediabetes and the global prevalence is projected to be 548 million (8.6\%) by 2045 [3]. Prediabetes has an annual conversion rate of $5-10 \%$ to T2DM [2, 4]. According to American Diabetes Association expert panel, up to $70 \%$ of individuals with prediabetes will eventually develop T2DM [1].

Whilst there is unequivocal evidence that many individuals with prediabetes are able to prevent the conversion to T2DM through lifestyle and diet modification [5-8], research is scarce regarding the use of complementary medicines and natural plant extracts to treat prediabetes. Studies of dietary polyphenols from natural plant sources have shown that some could have hypoglycaemic potential [9-13]. Proposed mechanisms by which they can improve glucose metabolism include the inhibition of digestive enzymes ( $\alpha$-amylase and $\alpha$ glucosidase) and glucose transporters, activation of glucokinase, increase in adiponectin levels, and elevation of incretin levels to stimulate glucosedependent insulin secretion [9-10,14-15]. Hence, polyphenol-rich plant extracts might be a useful adjunct in the preventing progression of prediabetes to T2DM.

Clinical trials conducted on healthy individuals and those with metabolic syndrome and T2DM have shown equivocal results regarding the consumption of grape seed [16-25], rooibos tea [25-26], and olive leaf extracts [27-34] likely due to concomitant medications, small sample sizes, as well as the plant extract sources, degree of purification and varying concentrations of extract administered in the trials. More importantly the inherent differences in metabolic profiles of participants might have likely contributed to the differences observed in the studies.

Indeed it is increasingly recognized that individuals with prediabetes are not a homogenous group and that differences in metabolic profiles identified as having different postprandial glucose shapes [35-37], postprandial glucose indices [36,38-41], and patterns of insulin concentrations [42-44] have been associated with varying degrees of risk towards T2DM. Therefore, stratification based on the different metabolic profiles is important in order to elucidate the impact of intervention on both responders and non-responders [45-47]. This would allow the opportunity for optimization of nutritional interventions for each specific metabolic profile.

More recently, Takahashi and colleagues (2018) examined the stratification based on the time to peak in glucose and insulin responses to differentiate between heterogeneous metabolic profiles of participants [48]. They classified participants into four groups: (1) normal type, (2) insulin-late type, (3) insulin- and glucose-late type, and (4) insulin-very late type [48]. They observed that whilst the first two groups (1) and (2) were more metabolically similar and had no significant differences in homeostatic model assessment-insulin resistance (HOMA-IR), homeostatic model assessment-beta-cell function (HOMA- $\beta$ ), area under the curve of glucose 
$\left(A \cup C_{\text {glucose }}\right)$, and area under the curve of insulin $\left(A \cup C_{\text {insulin }}\right)$. Groups (3) and (4) showed distinctly delayed insulin secretory responses and elevated $A \cup C_{\text {glucose }}$ compared to groups (1) and (2). Other studies have corroborated that a delay in glucose peak time has been used as a reproducible index for T2DM risk and a value of above $30 \mathrm{~min}$ indicates presence of insulin resistance and poorer glycemic control [39-40,49]. Insulin patterns such as delayed insulin response have been linked to defects in $\beta$-cell function and diminished insulin sensitivity in individuals and have been associated with an increased risk of developing T2DM $[42,44,50-52]$.

In our previous work we have shown that antioxidant-rich plant extracts such as the grape seed and rooibos tea extracts had helped to improve acute postprandial blood glucose response by $25-40 \%$ in healthy participants [25]. A chronic study conducted by de Bock and colleagues (2013) also demonstrated that with olive leaf insulin sensitivity and pancreatic $\beta$-cell responsiveness were significantly improved in normoglycemic, overweight men [29]. To date no study has investigated the hypoglycemic potential of these plant extracts in a group with prediabetes.

The present GLARE (Glucose Lowering AntioxidantRich plant Extracts) study aimed to examine the acute impact of grape seed, rooibos tea and olive leaf extracts on postprandial blood glucose response in individuals with prediabetes living in New Zealand. In addition, it will also explore if participants may be stratified into healthier subgroup similar to groups (1) and (2) and a less healthy subgroup similar to groups (3) and (4) after the Takahashi and colleagues (2018) classification, in order to see if responses to the intervention differ based on participants' prediabetic metabolic profile.

\section{METHODS}

Study participants: Participants were recruited from Auckland, New Zealand using poster advertisements within the local university and community (August 2017May 2019). They were selected if they met all inclusion criteria at screening: (1) having prediabetes with a HbA1c between $41-49 \mathrm{mmol} / \mathrm{mol}(5.9-6.6 \%)$ or a $\mathrm{HbA} 1 \mathrm{c}$ of $38-40 \mathrm{mmol} / \mathrm{mol}(5.6-5.8 \%)$ and having at least one of the T2DM risk factors (overweight (BMI $\geq 25 \mathrm{~kg} / \mathrm{m}^{2}$ ) or obese (BMI $\left.\geq 30 \mathrm{~kg} / \mathrm{m}^{2}\right)$, high blood pressure $(>120 / 80$ $\mathrm{mmHg}$ ), had prediabetes before, and family history of T2DM or cardiovascular disease (CVD)), (2) age between 40-80 years, (3) BMI between $18.5-40 \mathrm{~kg} / \mathrm{m}^{2}$, (4) not taking any form of glucose-lowering medications or medications having an iatrogenic nature that may influence glucose metabolism, particularly $\beta$-blockers for cardiovascular diseases and thiazide diuretics for hypertension, (5) no known pancreatic, hepatic, renal or digestive impairments that may alter glucose metabolism or metabolism of plant extracts, (6) no known allergies to the plant extracts under study, and (7) not smoking.

Interested individuals initially completed an online screening questionnaire on T2DM risk [53] before potential participants were invited into Massey University Human Nutrition Research Centre (MUHNRC) in Auckland for more in-depth screening and measurement of HbA1c (Cobas b $101 \mathrm{HbA1c}$ test, CV 0.81.7\%, Roche Diagnostics). 
All participants gave their informed consent for inclusion before they participated in the study. The study was conducted in accordance with the Declaration of Helsinki, and the protocol was approved by the Massey University Human Ethics Committee (MUHEC) (ref: 17/STH/82). The clinical trial was registered prospectively at the Australian New Zealand Clinical Trials Registry and accessible at

\section{http://www.anzctr.org.au/ (ACTRN12617000837325).}

Study design: The study was an acute, single-blind, placebo-controlled, non-randomized, crossover study involving 19 participants. The study involved four visits where participants were required to fast for at least ten hours except water before each visit.

At each trial visit, all participants were checked for dietary and lifestyle compliance, such as having fasted at least 10 hours prior to the visit, keeping their diet constant, no strenuous physical activity, no alcohol, no caffeinated tea or coffee formulations, no health supplements 24 hours to visit, and no consumption of active plant extracts (grape seed, rooibos tea, olive leaf) throughout the duration of the study. No participant had notable changes to their diet or lifestyle during the course of the study.

During the control (placebo) visit, participants were given two placebo capsules to swallow with a few sips of water. This was followed by a $2 \mathrm{~h}$ oral glucose tolerance test (OGTT) involving consuming a $300 \mathrm{~mL}$ glucose drink containing 75 g of glucose (Carbotest, Fronine, Thermo Fisher Scientific, Australia) within five minutes, and repeated measures of blood glucose during the following two hours. The subsequent three visits involved consuming one of the three plant extracts in the following order: grape seed, rooibos tea, and olive leaf on separate visits with at least a washout period of one week between visits. The participants did not know what treatment, or in which order the treatment was given. The OGTT procedure was repeated for each visit. All three plant extracts were matched for their total antioxidant capacity and administered in standardised capsule form. Antioxidant capacity has been shown in previous research to correlate with total phenolics [54, 55], and hence a good indicator of overall phenolic composition within each plant extract.

At each visit baseline venous blood samples were taken at $10 \mathrm{~min}$ prior to the consumption of the capsules, followed by another baseline sampling at $\mathrm{t}=0 \mathrm{~min}$ before the commencement of the OGTT. Further blood samples were obtained at $t=15,30,45,60,90$, and $120 \mathrm{~min}$. Blood draws were done by having the participant lie in a supine position and cannulating the antecubital fossa region of the arm. Blood collected in heparin tubes were immediately centrifuged after collection, whilst blood collected in serum tubes were left to coagulate for at least half an hour before centrifuging. They were then aliquot into Eppendorf tubes and stored at $-80^{\circ} \mathrm{C}$ until analysis.

Treatments: All the plant extract samples were obtained commercially. Grape seed extract (Vitis vinifera) (NutraLife, Vitaco Health (NZ) Ltd.) was produced with $40 \mathrm{~g}$ of dry grape seed and $10 \mathrm{~g}$ of fresh grape seed per capsule and contained $640 \mathrm{mg}$ of procyanidins in the concentration given to participants. Rooibos tea extract (Aspalathus linearis, E2CCJ) (Rooibos Limited, South 
Africa) contained at least $485 \mathrm{mg}$ of total polyphenols in the concentration given to participants. Olive leaf extract (Olea europaea) (Comvita NZ Ltd) was made from $3.5 \mathrm{~g}$ of fresh leaf suspended in olive oil per capsule and contained $264 \mathrm{mg}$ of oleuropeins in the concentration given to participants. The plant extracts were commercialised products and had no known adverse toxic effects associated with them or similar products as the levels consumed [56-58]. All extracts were prepared according to good manufacturing practice (GMP). The placebo capsule was made of 500 mg microcrystalline cellulose.

Sample extracts of grape seed, rooibos tea, and olive leaf were sent to Callaghan Innovation (Wellington, New Zealand) to determine their total antioxidant capacity (TAC) using the Oxygen Radical Absorbance Capacity (ORAC) assay. Following a previous study, but with some modifications [59], the ORAC assay was carried out with each sample of plant extract dissolved in a ratio of ethanol: water mixture $(70: 30, v / v)$ and their TAC determined.

Extracts of grape seed, rooibos tea, and olive leaf were matched at similar TAC content at 8,499 trolox equivalent (TE) $\mu \mathrm{mol}, 8,496 \mathrm{TE} \mu \mathrm{mol}$, and 9,152 TE $\mu \mathrm{mol}$, respectively, with concentrations based on a previous study [25]. Due to the nature of the olive leaf extract that was suspended in oil in a soft-gel and packed in a hard casing, contents could not be modified to obtain closer TAC values with grape seed and rooibos tea extracts.

\section{Measures of glucose and insulin responses during the} OGTT: The primary outcome of this study was incremental area under the curve of glucose (iAUC $C_{\text {glucose }}$ ).
The mean iAUC of the blood glucose (iAUC $C_{\text {glucose }}$ ) and insulin ( $\mathrm{iAUC}_{\text {insulin }}$ ) were analyzed using the trapezoidal rule $[60,61]$. Other indices of glucose such as $2 \mathrm{~h}$ postprandial glucose value (2hPG), and glucose peak time were measured. Time to glucose peak was defined as the time point on the OGTT when the glucose level was highest. If two equal peaks occurred during the OGTT, the earlier peak was considered as the peak. Similarly, $2 \mathrm{~h}$ postprandial insulin $(2 \mathrm{hPI})$, and insulin peak time were calculated. Insulin sensitivity indices such as the Matsuda Insulin Sensitivity Index (ISI/M) [62],

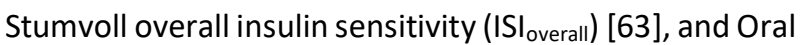
Glucose Insulin Sensitivity (OGIS) [64] were calculated. The insulin early phase responses were captured by insulinogenic index $\left(\mathrm{IGI}_{30}\right)[65]$ and Stumvoll first (ISI $\left.\right|_{\text {first }}$ ), whilst the late phase by Stumvoll second phase (ISI $\left.\right|_{\text {second }}$ ) insulin sensitivity indices [63]. The pancreatic $\beta$-cell function was calculated using the Insulin SecretionSensitivity Index-2 (ISSI-2) [66] and oral disposition index (DI), which was calculated as ratio of $\mathrm{IGI}_{30}$ to fasting insulin [67]. The metabolic clearance rate of glucose $\left(\mathrm{MCR}_{\text {glucose }}\right)$ was also calculated [63].

Analysis of blood samples: Plasma glucose and insulin samples were measured in a commercial laboratory (Waitemata District Health Board North Shore Hospital, Auckland, New Zealand). Plasma glucose concentrations were measured by the hexokinase method [68] (Vista GLU Flex ${ }^{\circledR}$ reagent cartridge, total CV 2-3\%, Dimension Vista ${ }^{\circledR} 1500$ System, Siemens Healthcare Limited). Plasma insulin was measured by a two-site sandwich immunoassay using direct chemiluminescent technology [69] (ADVIA Centaur Insulin assay, total CV 6.3-7.5\%, 
Siemens ADVIA Centaur XP, Siemens Healthcare Limited).

Sample size calculation and statistical analysis: A sample size calculation was performed based on the study done by Moore and colleagues (2000 and 2001) with an effect reduction of $100 \mathrm{mmol} . \mathrm{min} / \mathrm{L}$ targeted and hence at least 16 participants were required $[70,71]$. Statistical analyses were performed using IBM SPSS statistics version 27 (IBM corporation, New York, US). A linear mixed model for repeated measures using the repeated covariance compound symmetry with estimation employing restricted maximum likelihood (REML). A $p$ value of $\leq 0.05$ was considered to be significant ( $95 \%$ confidence level), based on $80 \%$ power with alpha $=0.05$ and beta $=0.10$. The data are expressed as model-adjusted mean \pm standard error of the mean (SEM).

\section{Secondary analysis of participants stratified into two} prediabetes subgroups: As impaired glycemic control involves a set of heterogeneous disorders [47], exploratory, secondary analysis was performed where participants were stratified into two prediabetes subgroups following the Takahashi and colleagues (2018) stratification method based on time to glucose and insulin peaks [48]. Participants in each subgroup would have more similar metabolic profiles. The healthy subgroup would have metabolic profiles that were distinctly different from the less healthy subgroup. This would help to elucidate the impact of plant extracts on glycemic responses in each prediabetes subgroup.
The healthier subgroup $(n=10)$ consisted of (1) normal type: participants with both normal glucose and insulin peaks (30 $\mathrm{min})$ and (2) insulin-late type: participants with normal glucose peak (30 min) but a delayed insulin peak (60 $\mathrm{min})$.

The less healthy subgroup $(n=9)$ consisted of (3) insulin- and glucose-late type: participants with both delayed glucose (60 $\mathrm{min}$ ) and delayed insulin peaks ( $\geq 60$ $\min )$, and (4) insulin-very late type: participants with normal glucose peak (30 $\mathrm{min}$ ) but very delayed insulin peaks (120 $\mathrm{min})$.

Statistical calculation was conducted similarly to the primary analysis. Differences between the two subgroups were analyzed using unpaired independent Student t-test assuming equal variances.

\section{RESULTS}

Nineteen participants with prediabetes (five men and fourteen women) completed the study (Figure 1). The overall mean age of the participants was $65.0 \pm 1.6$ years of age, with a mean BMI of $27.3 \pm 1.1 \mathrm{~kg} / \mathrm{m}^{2}$, and mean $\mathrm{HbA} 1 \mathrm{c}$ value of $42 \pm 1 \mathrm{mmol} / \mathrm{mol}$. Participant demographics for the complete group and after stratification are reported in Table 1 . There were no adverse events that occurred during the study trial. The plant extracts at their given doses were observed to be well-tolerated by all the participants.

At the control (placebo) visit, participants in the healthier subgroup had significantly lower $1 \mathrm{~h}$ and $2 \mathrm{~h}$ postprandial glucose $(p<0.05)$ and shorter glucose peak time $(p=0.017)$, lower $2 \mathrm{hPI}(p=0.022)$ and shorter insulin peak time $(p=0.002)$, higher insulin sensitivity $(p<0.05)$ and higher early-phase insulin sensitivity $(p<0.05)$, 
higher $\beta$-cell function $(p<0.05)$, as well as higher MCR glucose $_{(p=0.004)}$ compared to the less healthy subgroup. The significant differences in glucose and insulin levels, as well as insulin sensitivity justify the existence of two distinct metabolic groups in the participants.

Seven out of 19 participants had other accompanying comorbidities such as hypertension, high cholesterol and heart disease and were taking medications on a consistent basis. These medications were assessed to have no to minimal influence on glucose metabolism.

All nineteen participants completed both the control (placebo) and grape seed trials, whilst only 17 of them completed the subsequent rooibos tea and olive leaf trials, as two participants were withdrawn from the study at study visit 3 due to problems with obtaining enough blood via cannulation (Figure 1).

\section{Plant extracts and their impact on overall glucose and} insulin indices in participants prior to stratification into healthier and less healthy prediabetes subgroups: The impact of each plant extract (grape seed, rooibos tea and olive leaf) on glucose and insulin responses in comparison to placebo are shown in Table 2. Overall, there were no significant differences in glucose and insulin indices between plant extracts and placebo, nor amongst the plant extracts prior to stratifying into the prediabetes subgroups ( $p>0.05)$.
Plant extracts and their impact on glucose and insulin indices after stratification of participants into healthier and less healthy prediabetes subgroups Figure 2 and Table 3: show the postprandial glucose and insulin responses of the participants in the healthier and less healthy subgroups after stratification comparing each treatment with plant extract to the control (placebo).

In the less healthy subgroup, grape seed was shown to significantly reduce iAUC glucose $(p=0.016,21.9 \%$ reduction), $2 \mathrm{hPG}(p=0.034,14.7 \%$ reduction $), 2 \mathrm{hPI}$ $\left(p=0.029,22.4 \%\right.$ reduction). It also improved $\mathrm{ISI}_{\text {overall }}$ $(p=0.028,15.0 \%$ increase $)$ and MCR $_{\text {glucose }}(p=0.016$, $16.7 \%$ increase) compared to placebo. Rooibos tea extract significantly improved DI $(p=0.031,32.4 \%$ increase). Olive leaf extract was observed to significantly increase iAUC $C_{\text {insulin }}(p=0.040,16.7 \%$ increase).

In the healthier subgroup, a significantly higher $2 \mathrm{hPI}$ in the olive leaf extract treatment compared to placebo ( $p=0.030,49.5 \%$ increase) was observed. The higher insulin concentration inevitably led to a reduced $\mathrm{ISI}_{\text {overall }}(p=0.032,12.5 \%$ reduction $)$ and $\mathrm{MCR}_{\text {glucose }}$ ( $p=0.040,10.2 \%$ reduction).

Comparing between the healthier and less healthy subgroups, there were no significant differences in both glucose and insulin indices amongst the treatments $(p>0.05)$, except in the treatment with olive leaf extract, where $\mathrm{IGI}_{30}$ was significantly higher in the healthier subgroup than in the less healthy subgroup (1.6 \pm 0.4 vs $0.6 \pm 0.1, p=0.042)$. 


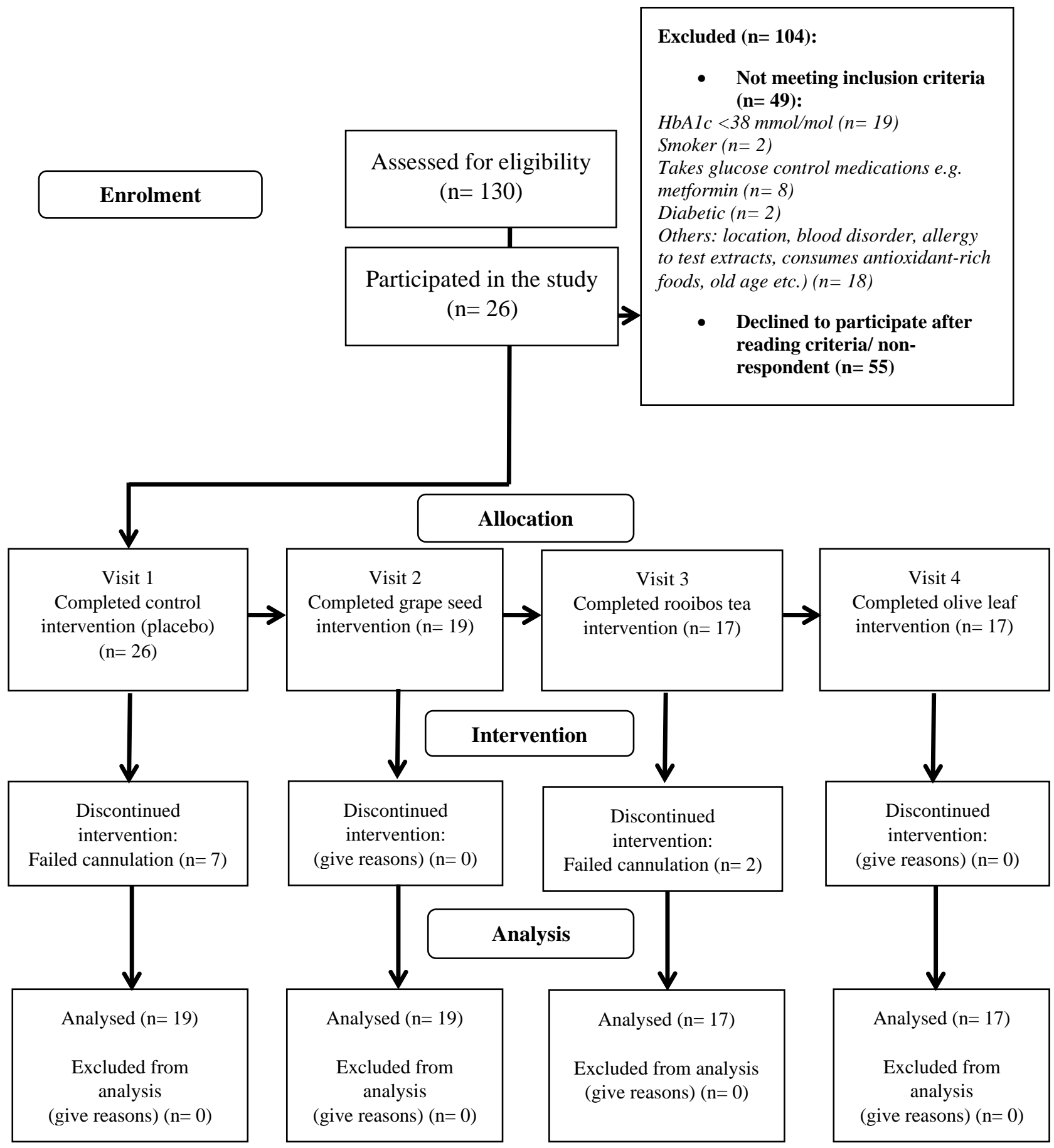

Figure 1. CONSORT flow diagram for the GLARE study. 
Table 1. Overall and stratified participant demographics at the control (placebo) visit.

\begin{tabular}{|c|c|c|c|c|}
\hline Characteristics & $\begin{array}{l}\text { Overall mean } \\
\text { ( } \pm \text { SEM) }\end{array}$ & $\begin{array}{l}\text { Healthier } \\
\text { subgroup mean } \\
\text { ( } \pm \text { SEM) }\end{array}$ & $\begin{array}{l}\text { Less healthy } \\
\text { subgroup mean } \\
\text { ( } \pm \text { SEM) }\end{array}$ & $\begin{array}{l}\text { p value between } \\
\text { healthier and less } \\
\text { healthy subgroups }\end{array}$ \\
\hline $\mathbf{N}$ & 19 & 10 & 9 & NA \\
\hline Gender (M/F) & $5 / 14$ & $2 / 8$ & $3 / 6$ & NA \\
\hline Age (years) & $65.0 \pm 1.6$ & $63.0 \pm 2.5$ & $67.2 \pm 1.9$ & 0.209 \\
\hline Body Mass Index, BMI (kg/m²) & $27.3 \pm 1.1$ & $26.0 \pm 1.7$ & $28.6 \pm 1.3$ & 0.249 \\
\hline Fasting blood glucose, FBG (mmol/L) & $5.7 \pm 0.1$ & $5.6 \pm 0.2$ & $5.9 \pm 0.2$ & 0.367 \\
\hline $\begin{array}{l}1 \mathrm{~h} \text { postprandial glucose, } 1 \mathrm{hPG} \\
(\mathrm{mmol} / \mathrm{L})\end{array}$ & $8.5 \pm 0.7$ & $6.6 \pm 0.5$ & $10.6 \pm 0.9$ & $0.001^{*}$ \\
\hline $\begin{array}{l}2 \mathrm{~h} \text { postprandial glucose, } 2 \mathrm{hPG} \\
(\mathrm{mmol} / \mathrm{L})\end{array}$ & $6.9 \pm 0.7$ & $5.0 \pm 0.3$ & $8.9 \pm 1.2$ & $0.003^{*}$ \\
\hline Fasting insulin, FI (mU/L) & $8.0 \pm 1.1$ & $7.2 \pm 1.2$ & $9.0 \pm 2.0$ & 0.418 \\
\hline $1 \mathrm{~h}$ postprandial insulin, $1 \mathrm{hPI}(\mathrm{mU} / \mathrm{L})$ & $70.5 \pm 9.2$ & $78.4 \pm 12.1$ & $61.8 \pm 14.2$ & 0.385 \\
\hline $2 \mathrm{~h}$ postprandial insulin, $2 \mathrm{hPI}(\mathrm{mU} / \mathrm{L})$ & $50.5 \pm 5.8$ & $38.6 \pm 6.5$ & $70.1 \pm 11.0$ & $0.022 *$ \\
\hline $\begin{array}{l}\text { Glycated hemoglobin A1c, HbA1c } \\
\text { (mmol/mol) }\end{array}$ & $42 \pm 1$ & $41 \pm 1$ & $43 \pm 1$ & 0.252 \\
\hline Systolic blood pressure $(\mathrm{mm} \mathrm{Hg})$ & $135 \pm 5$ & $130 \pm 5$ & $141 \pm 8$ & 0.237 \\
\hline Diastolic blood pressure (mm Hg) & $79 \pm 3$ & $79 \pm 5$ & $80 \pm 4$ & 0.919 \\
\hline
\end{tabular}

Values were presented as means \pm SEM. The healthier subgroup consisted of participants with both normal glucose and insulin peaks (30 $\mathrm{min}$ ) (normal type) and participants with normal glucose peak (30 min) but a delayed insulin peak (60 min) (insulin-late type). The less healthy subgroup consisted of participants with both delayed glucose (60 $\mathrm{min}$ ) and insulin peaks ( $\geq 60 \mathrm{~min}$ ) (insulin- and glucoselate type), and participants with normal glucose peak ( $30 \mathrm{~min}$ ) but very delayed insulin peaks (120 min) (insulin-very late type). *Signifies significant difference between healthier and less healthy subgroups, $p<0.05$ using the student t-test assuming equal variance. 
Table 2. Overall glycaemic outcomes of participants before stratification into two prediabetes subgroups.

\begin{tabular}{|c|c|c|c|c|c|}
\hline Analysis & Control (placebo) & Grape seed & Rooibos tea & Olive leaf & $\begin{array}{l}\text { Overall } p \\
\text { value }\end{array}$ \\
\hline \multicolumn{6}{|c|}{ Glucose indices } \\
\hline $\begin{array}{l}\text { Mean incremental area under the } \\
\text { curve of glucose, iAUC glucose } \\
\text { (mmol/L.min) }\end{array}$ & $266.4 \pm 46.3$ & $256.5 \pm 46.3$ & $286.2 \pm 46.8$ & $284.2 \pm 46.8$ & 0.605 \\
\hline $\begin{array}{l}2 \mathrm{~h} \text { postprandial glucose, } 2 \mathrm{hPG} \\
\text { (mmol/L) }\end{array}$ & $6.9 \pm 0.7$ & $6.6 \pm 0.7$ & $6.9 \pm 0.7$ & $7.0 \pm 0.7$ & 0.819 \\
\hline Glucose peak time (min) & $39.5 \pm 4.1$ & $43.4 \pm 4.1$ & $43.5 \pm 4.2$ & $36.8 \pm 4.2$ & 0.097 \\
\hline $\begin{array}{l}\text { Metabolic clearance rate of } \\
\text { glucose, } \text { MCR }_{\text {glucose }} \text { (mL/kg.min) }\end{array}$ & $7.5 \pm 0.6$ & $7.6 \pm 0.6$ & $7.5 \pm 0.6$ & $7.1 \pm 0.6$ & 0.307 \\
\hline \multicolumn{6}{|c|}{ Insulin sensitivity indices } \\
\hline Matsuda index & $5.3 \pm 0.7$ & $5.3 \pm 0.7$ & $5.4 \pm 0.7$ & $5.1 \pm 0.7$ & 0.870 \\
\hline $\begin{array}{l}\text { Oral glucose insulin sensitivity, } \\
\text { OGIS (mL/min. } \mathrm{m}^{2} \text { ) }\end{array}$ & $380.9 \pm 13.2$ & $379.3 \pm 13.2$ & $385.9 \pm 13.4$ & $377.4 \pm 13.4$ & 0.799 \\
\hline $\begin{array}{l}\text { Stumvoll overall insulin sensitivity } \\
\text { index, } \mathrm{ISI}_{\text {overall }}(\mathrm{pmol} / \mathrm{L})\end{array}$ & $0.08 \pm 0.01$ & $0.08 \pm 0.01$ & $0.08 \pm 0.01$ & $0.07 \pm 0.01$ & 0.153 \\
\hline \multicolumn{6}{|c|}{ Early and late phase insulin response and sensitivity indices } \\
\hline Insulinogenic index, $\mathbf{I G I}_{30}$ & $0.9 \pm 0.2$ & $1.0 \pm 0.2$ & $0.9 \pm 0.2$ & $1.0 \pm 0.2$ & 0.631 \\
\hline $\begin{array}{l}\text { Stumvoll first phase insulin } \\
\text { sensitivity index, ISI } \text { first }_{\text {(pmol/L) }}\end{array}$ & $1138.1 \pm 144.8$ & $1180.0 \pm 144.8$ & $1113.9 \pm 146.7$ & $1165.4 \pm 146.7$ & 0.865 \\
\hline $\begin{array}{l}\text { Stumvoll second phase insulin } \\
\text { sensitivity index, ISI } I_{\text {second }}(\mathrm{pmol} / \mathrm{L})\end{array}$ & $304.7 \pm 34.7$ & $314.6 \pm 34.7$ & $299.4 \pm 35.1$ & $311.8 \pm 35.1$ & 0.880 \\
\hline \multicolumn{6}{|l|}{$\beta$-cell function indices } \\
\hline $\begin{array}{l}\text { Insulin-secretion-sensitivity-index, } \\
\text { ISSI-2 }\end{array}$ & $34.0 \pm 3.5$ & $33.7 \pm 3.5$ & $33.7 \pm 3.6$ & $34.5 \pm 3.6$ & 0.995 \\
\hline $\begin{array}{l}\text { Oral disposition index, DI } \\
\text { (by insulin) }\end{array}$ & $0.12 \pm 0.03$ & $0.14 \pm 0.03$ & $0.13 \pm 0.03$ & $0.15 \pm 0.03$ & 0.684 \\
\hline \multicolumn{6}{|c|}{ Insulin secretion and response indices } \\
\hline $\begin{array}{l}\text { Mean incremental area under the } \\
\text { curve of insulin, iAUC } \text { insulin } \\
\text { (mU/L.min) }\end{array}$ & $5663.3 \pm 696.7$ & $5794.7 \pm 696.7$ & $5714.6 \pm 705.3$ & $6300.4 \pm 705.3$ & 0.354 \\
\hline $\begin{array}{l}2 \text { h postprandial insulin, } 2 \mathrm{hPI} \\
\text { (mU/L) }\end{array}$ & $53.5 \pm 7.1$ & $50.9 \pm 7.1$ & $51.4 \pm 7.3$ & $62.4 \pm 7.3$ & 0.153 \\
\hline Insulin peak time (min) & $75.8 \pm 6.4$ & $74.2 \pm 6.4$ & $82.9 \pm 6.7$ & $67.1 \pm 6.7$ & 0.154 \\
\hline
\end{tabular}


Table 3. Glycemic outcomes of participants in each prediabetes subgroup comparing plant extract treatment with control (placebo).

\begin{tabular}{|c|c|c|c|c|c|c|c|c|}
\hline \multirow[t]{2}{*}{ Glycemic measurements } & \multicolumn{4}{|c|}{ Healthier subgroup } & \multicolumn{4}{|c|}{ Less healthy subgroup } \\
\hline & Control (placebo) & Grape seed & Rooibos tea & Olive leaf & Control (placebo) & Grape seed & Rooibos tea & Olive leaf \\
\hline \multicolumn{9}{|c|}{ Glucose indices } \\
\hline $\begin{array}{l}\text { Mean incremental area under the curve } \\
\text { of glucose, iAUC glucose }(\mathrm{mmol} / \mathrm{L} \cdot \mathrm{min})\end{array}$ & $126.3 \pm 38.5$ & $190.8 \pm 38.5^{*}$ & $203.1 \pm 40.1^{*}$ & $171.0 \pm 40.1$ & $422.0 \pm 71.4$ & $329.5 \pm 71.4^{*}$ & $381.0 \pm 71.4$ & $405.7 \pm 71.4$ \\
\hline $2 \mathrm{~h}$ postprandial glucose, $2 \mathrm{hPG}(\mathrm{mmol} / \mathrm{L})$ & $5.0 \pm 0.5$ & $5.8 \pm 0.5$ & $5.4 \pm 0.5$ & $5.7 \pm 0.5$ & $8.9 \pm 1.2$ & $7.6 \pm 1.2^{*}$ & $8.4 \pm 1.2$ & $8.5 \pm 1.2$ \\
\hline Glucose peak time (min) & $31.5 \pm 3.2$ & $39.0 \pm 3.2$ & $34.2 \pm 3.5$ & $31.2 \pm 3.5$ & $48.3 \pm 7.3$ & $48.3 \pm 7.3$ & $53.3 \pm 7.3$ & $43.3 \pm 7.3$ \\
\hline $\begin{array}{l}\text { Metabolic clearance rate of glucose, } \\
\mathrm{MCR}_{\text {glucose }}(\mathrm{mL} / \mathrm{kg} \cdot \mathrm{min})\end{array}$ & $8.9 \pm 0.7$ & $8.4 \pm 0.7$ & $8.6 \pm 0.7$ & $8.0 \pm 0.7 *$ & $5.8 \pm 0.8$ & $6.8 \pm 0.8^{*}$ & $6.3 \pm 0.8$ & $6.0 \pm 0.8$ \\
\hline \multicolumn{9}{|c|}{ Insulin sensitivity indices } \\
\hline Matsuda index & $5.9 \pm 1.0$ & $5.7 \pm 1.0$ & $5.9 \pm 1.0$ & $5.9 \pm 1.0$ & $4.6 \pm 0.8$ & $4.7 \pm 0.8$ & $4.8 \pm 0.8$ & $4.2 \pm 0.8$ \\
\hline $\begin{array}{l}\text { Oral glucose insulin sensitivity, OGIS } \\
\left(\mathrm{mL} / \mathrm{min} \cdot \mathrm{m}^{2}\right)\end{array}$ & $405.4 \pm 16.8$ & $392.2 \pm 16.8$ & $410.9 \pm 17.7$ & $402.3 \pm 17.7$ & $353.7 \pm 18.3$ & $365.0 \pm 18.3$ & $360.0 \pm 18.3$ & $351.6 \pm 18.3$ \\
\hline $\begin{array}{l}\text { Stumvoll overall insulin sensitivity index, } \\
\text { ISI }_{\text {overall }}(\mathrm{pmol} / \mathrm{L} \text { ) }\end{array}$ & $0.09 \pm 0.01$ & $0.08 \pm 0.01$ & $0.09 \pm 0.01$ & $0.08 \pm 0.01^{*}$ & $0.06 \pm 0.01$ & $0.07 \pm 0.01^{*}$ & $0.07 \pm 0.01$ & $0.06 \pm 0.01$ \\
\hline \multicolumn{9}{|c|}{ Early and late phase insulin response and sensitivity indices } \\
\hline Insulinogenic index, $\mid \mathrm{GI}_{30}$ & $1.3 \pm 0.4$ & $1.4 \pm 0.4$ & $1.2 \pm 0.4$ & $1.6 \pm 0.4$ & $0.5 \pm 0.1$ & $0.6 \pm 0.1$ & $0.6 \pm 0.1$ & $0.6 \pm 0.1$ \\
\hline $\begin{array}{l}\text { Stumvoll first phase insulin sensitivity } \\
\text { index, ISI first }(\mathrm{pmol} / \mathrm{L})\end{array}$ & $1389.8 \pm 209.9$ & $1335.3 \pm 209.9$ & $1321.5 \pm 218.0$ & $1295.2 \pm 218.0$ & $858.4 \pm 185.4$ & $1007.5 \pm 185.4$ & $890.3 \pm 185.4$ & $1011.1 \pm 185.4$ \\
\hline $\begin{array}{l}\text { Stumvoll second phase insulin sensitivity } \\
\text { index, }||_{\text {second }}(\mathrm{pmol} / \mathrm{L})\end{array}$ & $362.2 \pm 50.3$ & $351.2 \pm 50.3$ & $348.6 \pm 52.4$ & $339.5 \pm 52.4$ & $240.8 \pm 44.9$ & $273.9 \pm 44.9$ & $246.9 \pm 44.9$ & $278.4 \pm 44.9$ \\
\hline \multicolumn{9}{|c|}{$\beta$-cell function indices } \\
\hline Insulin-secretion-sensitivity-index, ISSI-2 & $43.7 \pm 4.4$ & $41.5 \pm 4.4$ & $38.9 \pm 4.6$ & $42.6 \pm 4.6$ & $23.2 \pm 4.2$ & $25.0 \pm 4.2$ & $27.4 \pm 4.2$ & $25.6 \pm 4.2$ \\
\hline Oral disposition index, DI (by insulin) & $0.18 \pm 0.04$ & $0.20 \pm 0.04$ & $0.16 \pm 0.05$ & $0.22 \pm 0.05$ & $0.07 \pm 0.02$ & $0.08 \pm 0.02$ & $0.09 \pm 0.02 *$ & $0.08 \pm 0.02$ \\
\hline \multicolumn{9}{|c|}{ Insulin secretion and response indices } \\
\hline $\begin{array}{l}\text { Mean incremental area under the curve } \\
\text { of insulin, } i A U C_{\text {insulin }}(\mathrm{mU} / \mathrm{L} \cdot \mathrm{min})\end{array}$ & $5857.1 \pm 991.3$ & $6352.2 \pm 991.3$ & $5935.7 \pm 1024.6$ & $6158.6 \pm 1024.6$ & $5448.0 \pm 1031.8$ & $5175.2 \pm 1031.8$ & $5451.0 \pm 1031.8$ & $\begin{array}{l}6359.3 \pm \\
1031.8^{*}\end{array}$ \\
\hline $2 \mathrm{~h}$ postprandial insulin, $2 \mathrm{hPI}(\mathrm{mU} / \mathrm{L})$ & $38.6 \pm 8.4$ & $47.8 \pm 8.4$ & $42.8 \pm 9.0$ & $57.8 \pm 9.0^{*}$ & $70.1 \pm 11.3$ & $54.4 \pm 11.3^{*}$ & $60.8 \pm 11.3$ & $68.4 \pm 11.3$ \\
\hline Insulin peak time (min) & $57.0 \pm 7.5$ & $69.0 \pm 7.5$ & $64.7 \pm 8.3$ & $49.7 \pm 8.3$ & $96.7 \pm 7.7$ & $80.0 \pm 7.7$ & $100.0 \pm 7.7$ & $83.3 \pm 7.7$ \\
\hline
\end{tabular}

Values were adjusted means \pm SEM based on linear mixed model using compound symmetry as repeated covariance with restricted maximum likelihood (REML) estimation. The healthie

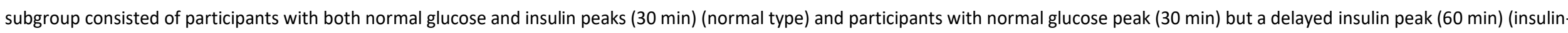

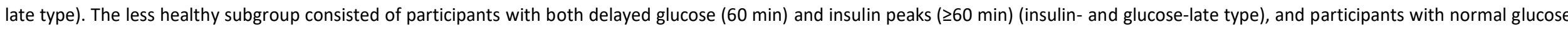
peak (30 $\mathrm{min}$ ) but very delayed insulin peaks $\left(120 \mathrm{~min}\right.$ ) (insulin-very late type). ${ }^{*}$ indicates significant difference $(p<0.05)$ between treatment and placebo. 


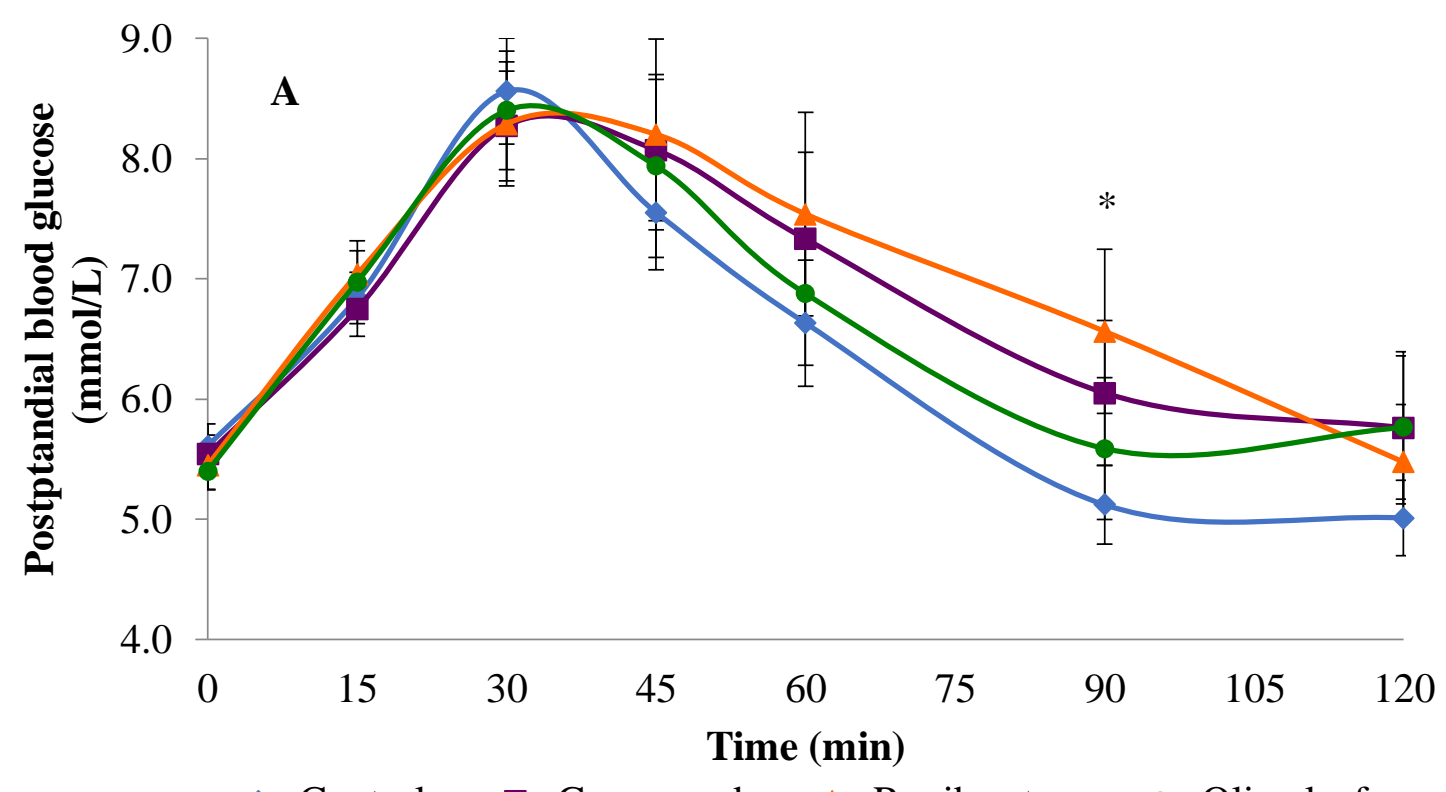

$\rightarrow$ Control $\rightarrow$ Grape seed $\rightarrow$ Rooibos tea $\rightarrow$ Olive leaf

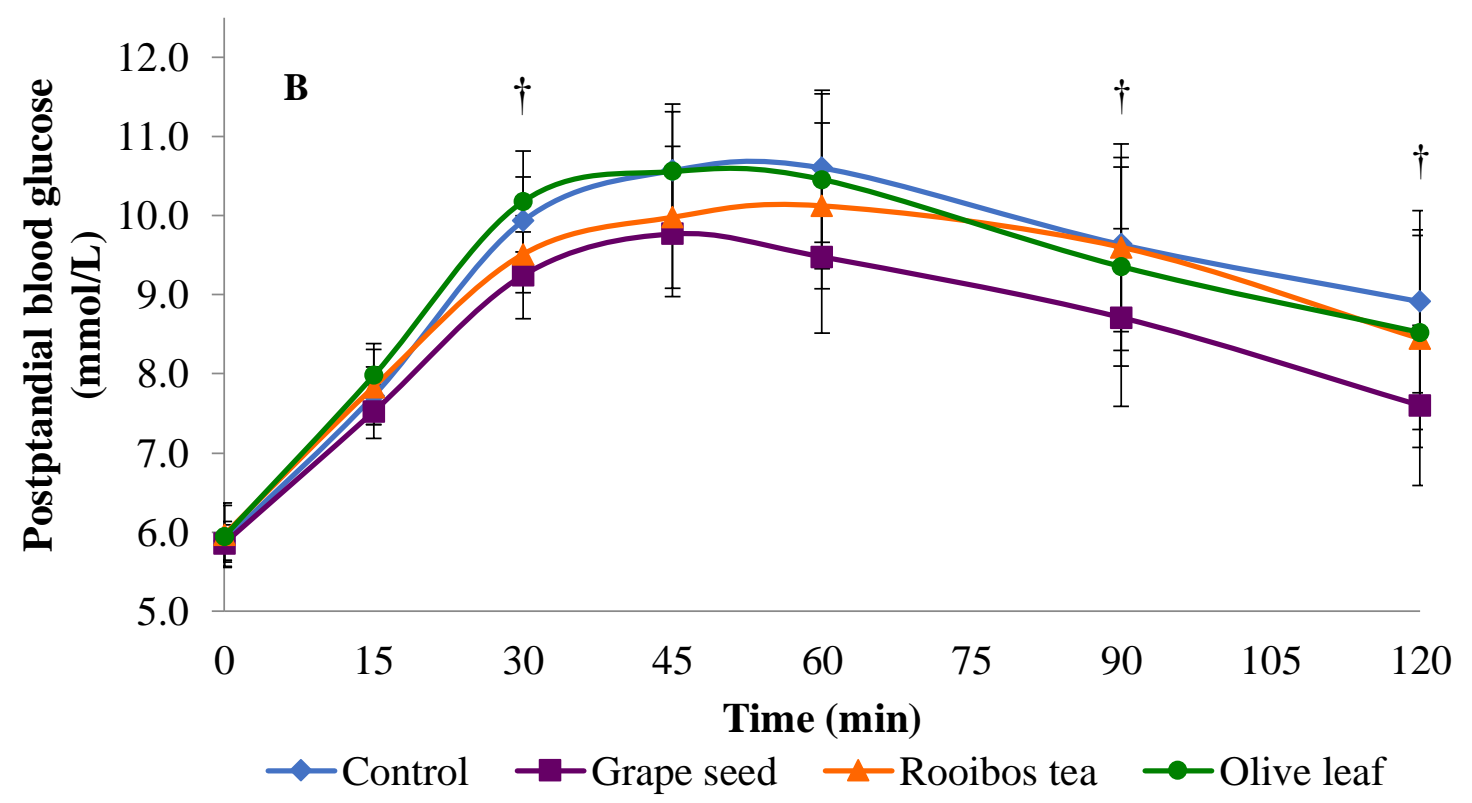

Figure 2. title and description continuing on the next page 

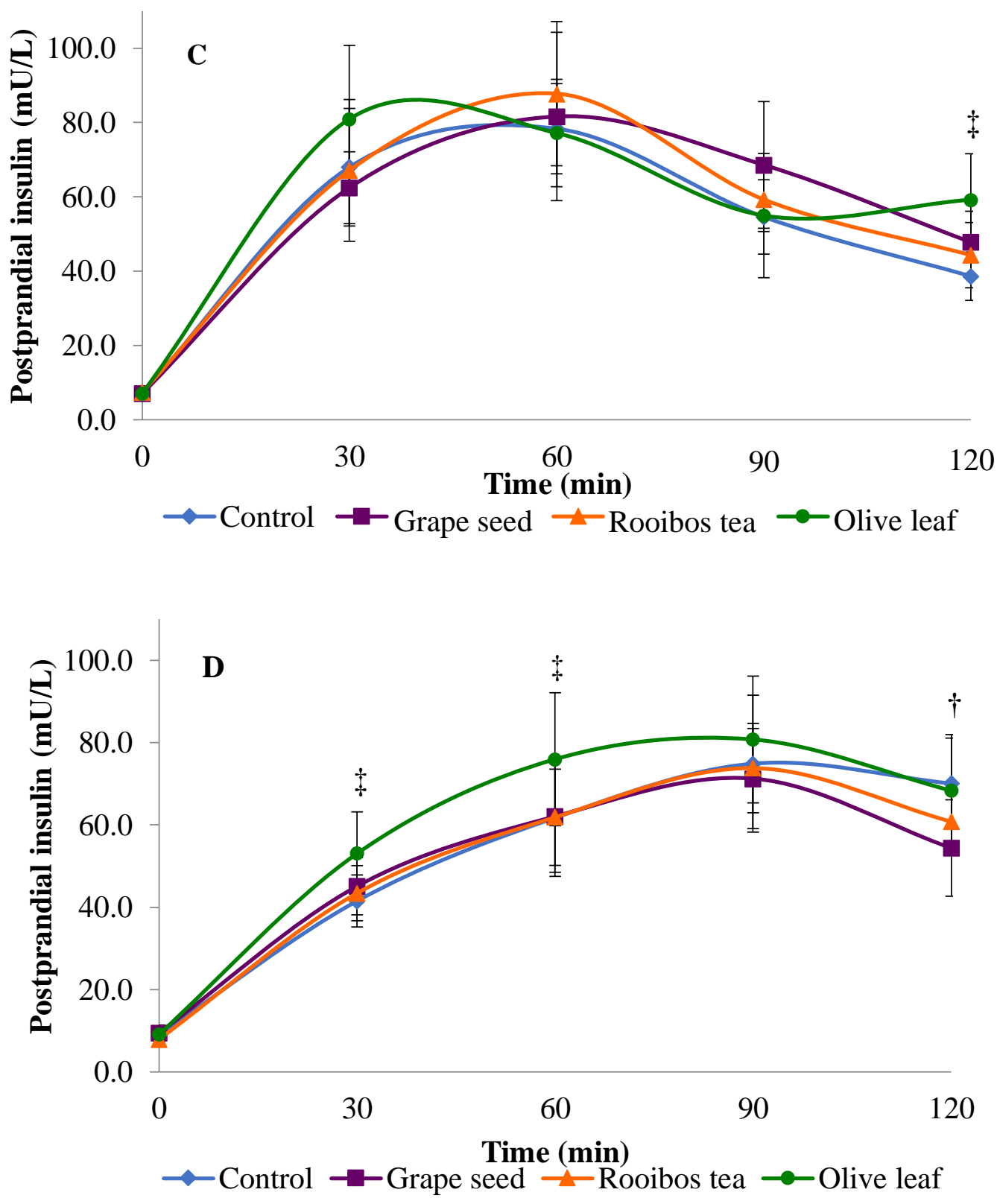

Figure 2. Unadjusted mean postprandial plasma glucose and insulin of participants during an oral glucose tolerance test (OGTT) with control (placebo), grape seed, rooibos tea and olive leaf extracts. (A) Postprandial glucose of participants in the healthier subgroup. (B) Postprandial glucose of participants in the less healthy subgroup. (C) Postprandial insulin of participants in the healthier subgroup. (D) Postprandial insulin of participants in the less healthy subgroup. The healthier subgroup consisted of participants with both normal glucose and insulin peaks (30 $\mathrm{min}$ ) (normal type) and participants with normal glucose peak (30 $\mathrm{min}$ ) but a delayed insulin peak (60 $\mathrm{min}$ ) (insulin-late type). The less healthy subgroup consisted of participants with both delayed glucose (60 $\mathrm{min}$ ) and insulin peaks ( $\geq 60 \mathrm{~min}$ ) (insulin- and glucose-late type), and participants with normal glucose peak (30 min) but very delayed insulin peaks (120 min) (insulin-very late type). Linear mixed model for repeated measures with repeated covariance compound symmetry with restricted maximum likelihood (REML) estimation was used to derive statistical significance. ${ }^{*}$ Indicates treatment with rooibos tea extract was significantly higher than placebo $(p<0.05) .{ }^{+}$indicates treatment with grape seed extract was significantly lower than placebo $(p<0.05) .{ }^{\ddagger}$ indicates treatment with olive leaf extract was significantly higher than placebo $(p<0.05)$. 


\section{DISCUSSION}

To the best of our knowledge the present study is the first to investigate the hypoglycemic impact of grape seed, rooibos tea, and olive leaf extracts in a prediabetes cohort. No significant results were seen for the group as a whole. However, when we stratified the GLARE study participants into two distinct metabolic subgroups (healthier versus less healthy subgroup) based on the work by Takahashi and colleagues (2018) [48] we observed differences in responses to the intervention.

In the less healthy subgroup, grape seed extract was shown to be the most effective in improving glucose and insulin responses compared to rooibos tea or olive leaf extract. Postprandial glucose and insulin concentrations were significantly reduced, whilst there were significant improvements in overall insulin sensitivity and $\mathrm{MCR}_{\text {glucose }}$ compared to placebo.

Observed differences in the healthier subgroup was an increased $\mathrm{i} A U \mathrm{C}_{\text {glucose }}$ with grape seed and rooibos tea extracts compared to placebo but nonetheless the iAUC $C_{\text {glucose }}$ values were significantly lower $(p<0.05)$ than that of the less healthy subgroup at the control visit. There was also an increase in $2 \mathrm{hPI}$ leading to reduced insulin sensitivity in the olive leaf extract compared to placebo due to the increased insulin response in the healthier subgroup.

The study has thus demonstrated that within the less healthy subgroup, participants were significantly more responsive to the intervention with plant extracts resulting in glycemic improvements, in contrast to the healthier subgroup. This observation was in corroboration with other research that elucidated heterogeneity in metabolic profiles could often lead to very different responses towards interventions and therefore stratification provides a platform for a more precise, targeted treatment [4547].

Acute, randomised controlled trials investigating grape seed extracts of various concentrations (100-500 mg) have shown reductions in $\mathrm{iACC}_{\text {glucose }}$ or $2 \mathrm{hPG}$ in healthy individuals and those with metabolic syndrome, in agreement with our findings $[19,23,25]$.

In the present study, rooibos tea extract was shown to significantly improve the DI indicating the possibility of potential improvement to the $\beta$-cell function in the less healthy subgroup. Restoring $\beta$-cell function is pivotal to restoring glucose homeostasis and delaying T2DM development [72-75]. Rooibos tea also showed improvements in iAUC $C_{\text {glucose }} \quad(9.7 \%$ reduction), $2 \mathrm{hPG}\left(5.2 \%\right.$ reduction) and $\mathrm{MCR}_{\text {glucose }}$ (8.4\% increase) in the less healthy subgroup compared to placebo, but these did not reach statistical significance $(p>0.05)$. This is in contrast to a previous clinical trial showed significant reduction in iAUC $_{\text {glucose }}(p<0.05,35.5 \%$ reduction $)$ in healthy participants after consuming $760 \mathrm{mg}$ of rooibos tea extract [25]. The difference in results might be due to the metabolic differences in the participants in the two studies and the higher dose used in the previous study.

Olive leaf extract was consistently shown to elevate insulin levels in the present study, with a higher $2 \mathrm{hPI}$ in the healthier subgroup and an elevated $\mathrm{iAUC}_{\text {insulin }}$ in the less healthy subgroup whilst having no significant reductions in postprandial glucose 
compared to placebo. The finding agrees with the acute study conducted by Kerimi and colleagues who showed no significant improvement in postprandial glucose in healthy participants after consuming olive leaf extract $(125-1000 \mathrm{mg}$ extract, $50-200 \mathrm{mg}$ oleuropein, respectively). Significant postprandial glucose reduction was only observed with $400 \mathrm{~g}$ of olive leaf extract (160 mg oleuropein) with a sucrose load [32]. In contrast, Komaki and colleagues (2003) showed that $1000 \mathrm{mg}$ of olive leaves consumed with $300 \mathrm{~g}$ of cooked rice in an acute study significantly reduced postprandial glucose at $30 \mathrm{~min}$ and $1 \mathrm{~h}$ in a borderline diabetic subgroup [27]. Prior research has demonstrated that olive leaf might be an insulin secretagogue and might be suitable for hyperglycaemic individuals secreting low levels of insulin due to impairment of the pancreatic $\beta$-cell function [76-77]. More research is warranted regarding the potential impact on glycaemia with olive leaf consumption.

Although the plant extracts tested were matched for total antioxidant capacity to allow for efficacy comparison as antioxidant capacity has been correlated to phenolic concentrations in several studies $[54,55]$, it was observed that the outcomes were not similar amongst the extracts. There is a possibility that the phenolic or antioxidant content of plant extracts might not fully correlate with the hypoglycemic actions exhibited [78-81]. Rather, the presence of different phenolic compositions in the extracts work collectively to structurally interact to inhibit digestive enzymes and glucose transporters including utilising several glucose metabolism pathways to impact glucose uptake and absorption [9-10, 14-15].
The strengths of the GLARE study included the use of a crossover design where participants were their own control resulting in a smaller sample size required. The plant extracts examined were also matched for TAC using the ORAC assay that is a wellresearched method, and allowed the comparison of efficacy amongst the extracts. Additionally, the present study has included a secondary analysis exploring two distinctly different prediabetes subgroups and using a number of glucose and insulin indices to quantify their responses to the plant extracts.

Nevertheless, the present study is not without limitations. Even though the exploratory, secondary analysis demonstrated observable glycemic improvements with the plant extracts, the sample size in each prediabetes subgroup was small. A larger sample size is thus recommended in future studies to elucidate the importance of considering prediabetes subgroups in nutrition interventions.

The current definition of prediabetes is somewhat arbitrary and is still expanding, and hence it is yet unclear whether it is truly a continuum or spectrum of heterogeneous worsening of glycemic control [82], or whether it is defined by distinct metabolic phenotypes [38]. Studies have elucidated the existence of prediabetes spectrum: IFG, IGT and IFG/IGT, and demonstrated them to be driven by different underlying dysfunctional metabolic profiles of glucose metabolism that included differences in glucose and insulin patterns $[83,84]$. The present study had recruited participants with prediabetes based solely on their $\mathrm{HbA} 1 \mathrm{c}$ values and not on these distinct metabolic phenotypes as a much larger sample size would be required.

The GLARE study consisted of participants who were only borderline prediabetic (mean HbA1c $42 \pm 1$ $\mathrm{mmol} / \mathrm{mol}$ ), and study outcomes observed in this 
study might not be replicable to those with worsening glycemic control and having a higher $\mathrm{HbA1c}$ reading closer to the diabetic range, and who may also be in greater need of intervention. However, a study conducted by Marini and colleagues (2014) on 338 non-diabetic offspring of T2DM parents using American Diabetes Association criteria for prediabetes revealed that those with $\mathrm{HbA1c}$ values above $39 \mathrm{mmol} / \mathrm{mol}(5.7 \%)$ already suffered from a significant faltering insulin sensitivity and $\beta$-cell function [85]. Edelman and colleagues (2004) in their research also recommended closer scrutiny for individuals having high-normal HbA1c (37.7-42.1 $\mathrm{mmol} / \mathrm{mol}, 5.6-6.0 \%)$ to elevated HbA1c (43.2-51.9 $\mathrm{mmol} / \mathrm{mol}, 6.1-6.9 \%)$ especially if they were obese because they had a higher diabetes incidence [86]. Therefore, it may be useful to consider intervention even for those with borderline prediabetes.

For future studies measurement of C-peptide would be more advantageous. C-peptide is cosecreted in equimolar amounts with insulin and does not undergo hepatic first pass prior to circulation unlike insulin and is not affected by impaired insulin clearance in insulin resistant individuals. It is therefore a better reflection of true insulin secretion [87-89].

\section{CONCLUSION}

Plant extracts have been increasingly gaining attention over the past decade as functional agents to improve glycaemic control. The current study has shown the potential acute hypoglycemic effects of grape seed, rooibos tea and olive leaf extracts and their impact on indices of glucose and insulin responses in individuals with prediabetes and having less healthy metabolic profiles. However, findings have been mixed likely due to differences in the extract types/grade, doses, sample sizes, and metabolic profile of participants between the present study and previous studies. Future studies should look into standardizing the extraction methods and concentrations of phenolic compounds in the plant extracts, and to investigate the relative impact of plant extracts on different metabolic profiles for a more targeted treatment. Where acute effects are observed, chronic studies are then needed to examine the longer-term impact of plant extracts in a prediabetes cohort.

List of Abbreviations: $\mathrm{AUC}_{\text {glucose: }}$ area under the curve of glucose, $A U C_{\text {insulin: }}$ area under the curve of insulin, BMI: body mass index, CVD: cardiovascular disease, DI: oral disposition index, FBG: fasting blood glucose, HbA1c: glycated hemoglobin A1c, HOMA-IR: homeostatic model of assessment-insulin resistance, HOMA- $\beta$ : homeostatic model of assessment-beta-cell function, $\mathrm{i} A \cup \mathrm{C}_{\text {glucose: }}$ incremental area under the curve

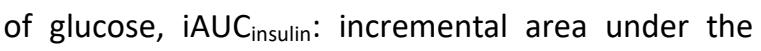
curve of insulin, IFG: impaired fasting glucose, $\mid \mathrm{IGI}_{30}$ : insulinogenic index, IGT: impaired glucose tolerance, IFG/IGT: combined impaired fasting glucose and impaired glucose tolerance, ISI/M: matsuda insulin

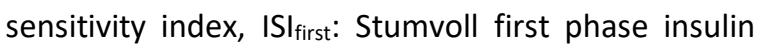

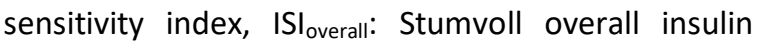

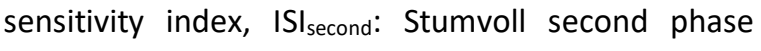
insulin sensitivity index, ISSI-2: insulin-secretionsensitivity-index-2, MCR glucose: metabolic clearance rate of glucose, NZSSD: New Zealand Society for the Study of Diabetes, OGIS: oral glucose insulin sensitivity, OGTT: oral glucose tolerance test, ORAC: oxygen radical absorbance capacity, TAC: total antioxidant capacity, TE: trolox equivalent, T2DM: type 2 diabetes mellitus, 1hPG: $1 \mathrm{~h}$ postprandial glucose, 2hPG: $2 \mathrm{~h}$ postprandial glucose, 1hPI: $2 \mathrm{~h}$ postprandial insulin, $2 \mathrm{hPI}: 2 \mathrm{~h}$ postprandial insulin. 
Competing Interests: The authors report no conflicts of interest.

Authors' Contributions: The authors' contributions were as follows - all authors: designed and provided oversight of the GLARE study; Owen Mugridge and Wen Xin Janice Lim: conducted the research; Wen Xin Janice Lim: analyzed the data, performed the statistical analyses, wrote the paper, and had primary

\section{REFERENCES}

1 Buysschaert M, Bergman M: Definition of prediabetes. Medical Clinics of North America 2011, 95(2):289-297. http://www.doi.org/10.1016/j.mcna.2010.11.002

2. Bansal N: Prediabetes diagnosis and treatment: A review. World Journal of Diabetes 2015, 6(2):296-303.

http://www.doi.org/10.4239/wjd.v6.i2.296

3. International Diabetes Federation: IDF Diabetes Atlas; 2019.

4. Gerstein HC, Santaguida P, Raina P, Morrison KM, Balion C, Hunt D, Yazdi H, Booker L: Annual incidence and relative risk of diabetes in people with various categories of dysglycemia: A systematic overview and meta-analysis of prospective studies. Diabetes Research and Clinical Practice 2007, 78(3):305-312.

\section{http://www.doi.org/10.1016/j.diabres.2007.05.004}

5. Perreault L, Pan Q, Mather KJ, Watson KE, Hamman RF, Kahn SE: Effect of regression from prediabetes to normal glucose regulation on long-term reduction in diabetes risk: results from the Diabetes Prevention Program Outcomes Study. Lancet 2012, 379(9833):2243-2251. http://www.doi.org/10.1016/s0140-6736(12)60525-x

6. Gong Q, Gregg EW, Wang J, An Y, Zhang P, Yang W, Li H, Li $H$, Jiang $Y$, Shuai $Y$, Zhang B, Zhang J, Gerzoff RB, Roglic G, Hu Y, Li G, Bennett PH: Long-term effects of a randomised trial of a 6-year lifestyle intervention in impaired glucose tolerance on diabetes-related microvascular complications: the China Da Qing Diabetes Prevention Outcome Study. Diabetologia 2011, 54(2):300-307. http://www.doi.org/10.1007/s00125-010-1948-9

7. Tabak AG, Herder C, Rathmann W, Brunner EJ, Kivimaki M: Prediabetes: a high-risk state for diabetes development. Lancet 2012, 379(9833):2279-2290. responsibility for the final content; all authors: have revised, read, and approved the final manuscript.

\section{Acknowledgements and Funding: Rooibos tea} extract powder was supplied by South Africa Rooibos Council, who also sponsored and funded part of the study involving the rooibos tea extract trial. They had no role in the design, analysis or writing of this article. The study was internally sponsored by Massey University, College of Health.

\section{http://www.doi.org/10.1016/s0140-6736(12)60283-9}

8. Li GW, Zhang P, Wang JP, Gregg EW, Yang WY, Gong QH, Li $H$, Li HL, Jiang YY, An YL, Shuai $Y$, Zhang B, Zhang JL, Thompson TJ, Gerzoff RB, Roglic G, Hu YH, Bennett PH: The long-term effect of lifestyle interventions to prevent diabetes in the China Da Qing Diabetes Prevention Study: a 20-year follow-up study. Lancet 2008, 371(9626):17831789.

http://www.doi.org/10.1016/s0140-6736(08)60766-7

9. Williamson G: Possible effects of dietary polyphenols on sugar absorption and digestion. Molecular Nutrition and Food Research 2013, 57(1):48-57. http://www.doi.org/10.1002/mnfr.201200511

10. Cheynier V: Polyphenols in foods are more complex than often thought. American Journal of Clinical Nutrition 2005, 81(1):223S-229S.

http://www.doi.org/10.1093/ajcn/81.1.223S

11. Burton-Freeman B, Brzezinski M, Park E, Sandhu A, Xiao D, Edirisinghe I: A selective role of dietary anthocyanins and flavan-3-ols in reducing the risk of type 2 diabetes mellitus: a review of recent evidence. Nutrients 2019, 11(4):1-16. http://www.doi.org/10.3390/nu11040841

12. Cao H, Ou JY, Chen L, Zhang YB, Szkudelski T, Delmas D, Daglia M, Xiao JB: Dietary polyphenols and type 2 diabetes: human study and clinical trial. Critical Reviews in Food Science and Nutrition 2019, 59(20):3371-3379. http://www.doi.org/10.1080/10408398.2018.1492900

13. Zhao C, Yang CF, Wai STC, Zhang YB, Portillo MP, Paoli P, Wu YJ, Cheang WS, Liu B, Carpene C, Xiao JB, Cao H: Regulation of glucose metabolism by bioactive phytochemicals for the management of type 2 diabetes mellitus. Critical Reviews in Food Science and Nutrition 2019, 59(6):830-847. 
http://www.doi.org/10.1080/10408398.2018.1501658

14. Scalbert A, Manach C, Morand C, Remesy C, Jimenez L: Dietary polyphenols and the prevention of diseases. Critical Reviews in Food Science and Nutrition 2005, 45(4):287-306.

http://www.doi.org/10.1080/1040869059096

15. Scalbert A, Johnson IT, Saltmarsh M: Polyphenols: antioxidants and beyond. American Journal of Clinical Nutrition 2005, 81(1):215S-217S. http://www.doi.org/10.1093/ajcn/81.1.215S

16. Kar $P$, Laight $D$, Rooprai HK, Shaw KM, Cummings $M$ : Effects of grape seed extract in Type 2 diabetic subjects at high cardiovascular risk: a double blind randomized placebo controlled trial examining metabolic markers, vascular tone, inflammation, oxidative stress and insulin sensitivity. Diabetic Medicine 2009, 26(5):526-531. http://www.doi.org/10.1111/j.14645491.2009.02727.x

17. Sivaprakasapillai B, Edirisinghe I, Randolph J, Steinberg F, Kappagoda T: Effect of grape seed extract on blood pressure in subjects with the metabolic syndrome. Metabolism-Clinical and Experimental 2009, 58(12):17431746.

http://www.doi.org/10.1016/j.metabol.2009.05.030

18. Pourghassem-Gargari B, Abedini S, Babaei H, Aliasgarzadeh A, Pourabdollahi P: Effect of supplementation with grape seed (Vitis vinifera) extract on antioxidant status and lipid peroxidation in patient with type II diabetes. Journal of Medicinal Plants Research 2011, 5(10):2029-2034.

19. Edirisinghe I, Randolph J, Cheema M, Tadapaneni R, Park E, Burton-Freeman B, Kappagoda T: Effect of grape seed extract on postprandial oxidative status and metabolic responses in men and women with the metabolic syndrome. Randomized, cross-over, placebo-controlled study. Functional Foods in Health and Disease 2012, 2(12):508-521.

http://www.doi.org/10.31989/ffhd.v2i12.68

20. Sano A, Uchida R, Saito M, Shioya N, Komori Y, Tho Y, Hashizume N: Beneficial effects of grape seed extract on malondialdehyde-modified LDL. Journal of Nutritional Science and Vitaminology 2007, 53(2):174-182. http://www.doi.org/10.3177/jnsv.53.174

21. Mellen PB, Daniel KR, Brosnihan KB, Hansen KJ, Herrington DM: Effect of muscadine grape seed supplementation on vascular function in subjects with or at risk for cardiovascular disease: a randomized crossover trial.
Journal of the American College of Nutrition 2010, 29(5):469-475.

http://www.doi.org/10.1080/07315724.2010.10719883

22. Robinson M, Lu B, Edirisinghe I, Kappagoda CT: Effect of grape seed extract on blood pressure in subjects with prehypertension. Journal of Pharmacy and Nutrition Sciences 2012, 2(2):155-159.

http://www.doi.org/10.6000/1927-5951.2012.02.02.6

23. Sapwarobol S, Adisakwattana S, Changpeng $S$, Ratanawachirin W, Tanruttanawong K, Boonyarit W: Postprandial blood glucose response to grape seed extract in healthy participants: A pilot study. Pharmacognosy Magazine 2012, 8(31):192-196. http://www.doi.org/10.4103/0973-1296.99283

24. Park E, Edirisinghe I, Choy YY, Waterhouse A, BurtonFreeman B: Effects of grape seed extract beverage on blood pressure and metabolic indices in individuals with pre-hypertension: a randomised, double-blinded, twoarm, parallel, placebo-controlled trial. British Journal of Nutrition 2016, 115(2):226-238. http://www.doi.org/10.1017/s0007114515004328

25. Chepulis L, Al-Aubaidy H, Page R: Effects of selected antioxidant food extracts on postprandial glucose responses in healthy individuals. Functional Foods in Health and Disease 2016, 6(8):493-505. http://www.doi.org/10.31989/ffhd.v6i8.271

26. Marnewick JL, Rautenbach F, Venter I, Neethling H, Blackhurst DM, Wolmarans P, Macharia M: Effects of rooibos (Aspalathus linearis) on oxidative stress and biochemical parameters in adults at risk for cardiovascular disease. Journal of Ethnopharmacology 2011, 133(1):4652. http://www.doi.org/10.1016/j.jep.2010.08.061

27. Komaki E, Yamaguchi S, Maru I, Kinoshita M, Kakehi K, Ohta $\mathrm{Y}$, Tsukada $\mathrm{Y}$ : Identification of anti-alpha-amylase components from olive leaf extracts. Food Science and Technology Research 2003, 9(1):35-39.

http://www.doi.org/10.3136/fstr.9.35

28. Wainstein J, Ganz T, Boaz M, Bar Dayan Y, Dolev E, Kerem Z, Madar Z: Olive leaf extract as a hypoglycemic agent in both human diabetic subjects and in rats. Journal of Medicinal Food 2012, 15(7):605-610. http://www.doi.org/10.1089/jmf.2011.0243

29. de Bock M, Derraik JGB, Brennan CM, Biggs JB, Morgan PE, Hodgkinson SC, Hofman PL, Cutfield WS: Olive (Olea europaea L.) Leaf polyphenols improve insulin sensitivity in 
middle-aged overweight men: a randomized, placebocontrolled, crossover trial. Plos One 2013, 8(3):1-8.

http://www.doi.org/10.1371/journal.pone.0057622

30. Wong RHX, Garg ML, Wood LG, Howe PRC: Antihypertensive potential of combined extracts of olive leaf, green coffee bean and beetroot: a randomized, double-blind, placebo-controlled crossover trial. Nutrients $2014,6(11): 4881-4894$.

http://www.doi.org/10.3390/nu6114881

31. Lockyer S, Rowland I, Spencer JPE, Yaqoob P, Stonehouse W: Impact of phenolic-rich olive leaf extract on blood pressure, plasma lipids and inflammatory markers: a randomised controlled trial. European Journal of Nutrition 2017, 56(4):1421-1432.

http://www.doi.org/10.1007/s00394-016-1188-y

32. Kerimi A, Nyambe-Silavwe H, Pyner A, Oladele E, Gauer JS, Stevens $Y$, Williamson G: Nutritional implications of olives and sugar: attenuation of post-prandial glucose spikes in healthy volunteers by inhibition of sucrose hydrolysis and glucose transport by oleuropein. European Journal of Nutrition 2018, 58(3):1315-1330.

http://www.doi.org/10.1007/s00394-018-1662-9

33. Araki R, Fujie $K$, Yuine N, Watabe $Y$, Nakata $Y$, Suzuki $H$, Isoda $\mathrm{H}$, Hashimoto $\mathrm{K}$ : Olive leaf tea is beneficial for lipid metabolism in adults with prediabetes: an exploratory randomized controlled trial. Nutrition Research 2019, 67:60-66.

http://www.doi.org/10.1016/j.nutres.2019.05.003

34. Pyner A, Chan SY, Tumova S, Kerimi A, Williamson G: Indirect chronic effects of an oleuropein-rich olive leaf extract on sucrase-isomaltase in vitro and in vivo. Nutrients 2019, 11(7):1-14. http://www.doi.org/10.3390/nu11071505

35. Tschritter O, Fritsche A, Shirkavand F, Machicao F, Haring $H$, Stumvoll M: Assessing the shape of the glucose curve during an oral glucose tolerance test. Diabetes Care 2003, 26(4):1026-1033.

\section{http://www.doi.org/10.2337/diacare.26.4.1026}

36. Kim JY, Michaliszyn SF, Nasr A, Lee S, Tfayli H, Hannon T, Hughan KS, Bacha F, Arslanian S: The shape of the glucose response curve during an oral glucose tolerance test heralds biomarkers of type 2 diabetes risk in obese youth. Diabetes Care 2016, 39(8):1431-1439.

http://www.doi.org/10.2337/dc16-0352

37. Kaga H, Tamura Y, Takeno K, Kakehi S, Someya Y, Suzuki R, Kadowaki S, Sugimoto D, Furukawa Y, Funayama T,
Kawamori $\mathrm{R}$, Watada $\mathrm{H}$ : The shape of the glucose response curveduring an oral glucose tolerance test was associated with muscle insulin sensitivity and visceral fat accumulation in nonobese healthy men. Diabetes 2018, 67(Suppl 1):1-2. http://www.doi.org/10.2337/db18-1512-P

38. Schianca GPC, Colli E, Onolfo S, Pedrazzoli R, Fra GP, Bartoli E: Individuation of different metabolic phenotypes in normal glucose tolerance test. Acta Diabetologica 2010, 47(2):167-172. http://www.doi.org/10.1007/s00592-009-0165-9

39. Kramer CK, Vuksan V, Choi H, Zinman B, Retnakaran R: Emerging parameters of the insulin and glucose response on the oral glucose tolerance test: Reproducibility and implications for glucose homeostasis in individuals with and without diabetes. Diabetes Research and Clinical Practice 2014, 105(1):88-95. http://www.doi.org/10.1016/j.diabres.2014.04.023

40. Chung ST, Ha J, Onuzuruike AU, Kasturi K, Galvan-De La Cruz M, Bingham BA, Baker RL, Utumatwishima JN, Mabundo LS, Ricks M, Sherman AS, Sumner AE: Time to glucose peak during an oral glucose tolerance test identifies prediabetes risk. Clinical Endocrinology 2017, 87(5):484-491. http://www.doi.org/10.1111/cen.13416

41. Hulman A, Vistisen D, Glumer C, Bergman M, Witte DR, Faerch K: Glucose patterns during an oral glucose tolerance test and associations with future diabetes, cardiovascular disease and all-cause mortality rate. Diabetologia 2018, 61(1):101-107. http://www.doi.org/10.1007/s00125-017-4468-z

42. Hayashi T, Boyko EJ, Sato KK, McNeely MJ, Leonetti DL, Kahn SE, Fujimoto WY: Patterns of insulin concentration during the OGTT predict the risk of type 2 diabetes in Japanese Americans. Diabetes Care 2013, 36(5):12291235.

http://www.doi.org/10.2337/dc12-0246

43. Crofts C, Schofield G, Zinn C, Wheldon M, Kraft J: Identifying hyperinsulinaemia in the absence of impaired glucose tolerance: An examination of the Kraft database. Diabetes Research and Clinical Practice 2016, 118:50-57. http://www.doi.org/10.1016/j.diabres.2016.06.007

44. Sun Y, Han JF, Lin ZW, Song LG, Wang C, Jia WP: Delayed insulin secretion response during an OGTT is associated with an increased risk for incidence of diabetes in NGT subjects. Journal of Diabetes and Its Complications 2016, 30(8):1537-1543. 
http://www.doi.org/10.1016/j.jdiacomp.2016.07.029

45. Morris C, O'Grada C, Ryan M, Roche HM, Gibney MJ, Gibney ER, Brennan L: Identification of differential responses to an oral glucose tolerance test in healthy adults. Plos One 2013, 8(8):1-9.

http://www.doi.org/10.1371/journal.pone.0072890

46. Krishnan S, Newman JW, Hembrooke TA, Keim NL: Variation in metabolic responses to meal challenges differing in glycemic index in healthy women: Is it meaningful? Nutrition and Metabolism 2012, 9(26):1-10. http://www.doi.org/10.1186/1743-7075-9-26

47. Faerch K, Hulman A, Solomon TPJ: Heterogeneity of prediabetes and type 2 diabetes: implications for prediction, prevention and treatment responsiveness. Current Diabetes Reviews 2016, 12(1):30-41. http://www.doi.org/10.2174/1573399811666150416122 $\underline{903}$

48. Takahashi K, Nakamura H, Sato H, Matsuda H, Takada K, Tsuji T: Four plasma glucose and insulin responses to a $75 \mathrm{~g}$ OGTT in healthy young Japanese women. Journal of Diabetes Research 2018:1-7.

http://www.doi.org/10.1155/2018/5742497

49. Wang XL, Zhao XQ, Zhou RR, Gu YJ, Zhu XH, Tang ZQ, Yuan $\mathrm{XL}$, Chen W, Zhang RP, Qian C, Cui SW: Delay in glucose peak time during the oral glucose tolerance test as an indicator of insulin resistance and insulin secretion in type 2 diabetes patients. Journal of Diabetes Investigation 2018, 9(6):1288-1295.

http://www.doi.org/10.1111/jdi.12834

50. DiNicolantonio JJ, Bhutani J, Okeefe JH, Crofts C: Postprandial insulin assay as the earliest biomarker for diagnosing pre-diabetes, type 2 diabetes and increased cardiovascular risk. Open Heart 2017, 4(2):1-4.

http://www.doi.org/10.1136/openhrt-2017-000656

51. Guillausseau PJ, Meas T, Virally $M$, Laloi-Michelin $M$, Medeau V, Kevorkian JP: Abnormalities in insulin secretion in type 2 diabetes mellitus. Diabetes and Metabolism 2008, 34(Suppl 2):S43-S48.

http://www.doi.org/10.1016/s1262-3636(08)73394-9

52. Festa A, Williams K, Hanley AJG, Haffner SM: beta-Cell dysfunction in subjects with impaired glucose tolerance and early type 2 diabetes - Comparison of surrogate markers with first-phase insulin secretion from an intravenous glucose tolerance test. Diabetes 2008, 57(6):1638-1644.

http://www.doi.org/10.2337/db07-0954
53. Ministry of Health: New Zealand Primary Care Handbook 2012. New Zealand; 2012.

54. Yemis O, Bakkalbasi E, Artik N: Antioxidative activities of grape (Vitis vinifera) seed extracts obtained from different varieties grown in Turkey. International Journal of Food Science and Technology 2008, 43(1):154-159.

http://www.doi.org/10.1111/j.1365-2621.2006.01415.x

55. Brewer MS: Natural antioxidants: sources, compounds, mechanisms of action, and potential applications. Comprehensive Reviews in Food Science and Food Safety 2011, 10(4):221-247.

http://www.doi.org/10.1111/j.1541-4337.2011.00156.x

56. Yamakoshi J, Saito M, Kataoka S, Kikuchi M: Safety evaluation of proanthocyanidin-rich extract from grape seeds. Food and Chemical Toxicology 2002, 40(5):599-607. http://www.doi.org/10.1016/s0278-6915(02)00006-6

57. Clewell AE, Beres E, Vertesi A, Glavits R, Hirka G, Endres JR, Murbach TS, Szakonyine IP: A comprehensive toxicological safety assessment of an extract of olea europaea L. leaves (Bonolive). International Journal of Toxicology 2016, 35(2):208-221.

http://www.doi.org/10.1177/1091581815619764

58. Joubert E, Gelderblom WCA, Louw A, de Beer D: South African herbal teas: Aspalathus linearis, Cyclopia spp. and Athrixia phylicoides-A review. Journal of Ethnopharmacology 2008, 119(3):376-412.

http://www.doi.org/10.1016/j.jep.2008.06.014

59. Zulueta A, Esteve MJ, Frigola A: ORAC and TEAC assays comparison to measure the antioxidant capacity of food products. Food Chemistry 2009, 114(1):310-316. http://www.doi.org/10.1016/j.foodchem.2008.09.033

60. Wolever TMS, Jenkins DJA: The use of the glycemic index in predicting the blood glucose response to mixed meals. American Journal of Clinical Nutrition 1986, 43(1):167-172. http://www.doi.org/10.1093/ajcn/43.1.167

61. Lefloch JP, Escuyer P, Baudin E, Baudon D, Perlemuter L: Blood glucose area under the curve: methodological aspects. Diabetes Care 1990, 13(2):172-175.

http://www.doi.org/10.2337/diacare.13.2.172

62. Matsuda M, DeFronzo RA: Insulin sensitivity indices obtained from oral glucose tolerance testing - Comparison with the euglycemic insulin clamp. Diabetes Care 1999, 22(9):1462-1470.

http://www.doi.org/10.2337/diacare.22.9.1462

63. Stumvoll M, Van Haeften T, Fritsche A, Gerich J: Oral glucose tolerance test indexes for insulin sensitivity and 
secretion based on various availabilities of sampling times. Diabetes Care 2001, 24(4):796-797.

http://www.doi.org/10.2337/diacare.24.4.796

64. Mari A, Pacini G, Murphy E, Ludvik B, Nolan JJ: A modelbased method for assessing insulin sensitivity from the oral glucose tolerance test. Diabetes Care 2001, 24(3):539-548. http://www.doi.org/10.2337/diacare.24.3.539

65. Phillips DI, Clark PM, Hales CN, Osmond C: Understanding oral glucose tolerance. Comparison of glucose or insulin measurements during the oral glucose tolerance test with specific measurements of insulin resistance and insulin secretion. Diabetic Medicine 1994, 11(3):286-292.

http://www.doi.org/10.1111/j.1464-5491.1994.tb00273.x

66. Retnakaran R, Shen S, Hanley AJ, Vuksan V, Hamilton JK, Zinman B: Hyperbolic relationship between insulin secretion and sensitivity on oral glucose tolerance test. Obesity 2008, 16(8):1901-1907.

http://www.doi.org/10.1038/oby.2008.307

67. Utzschneider KM, Prigeon RL, Faulenbach MV, Tong J, Carr DB, Boyko EJ, Leonetti DL, McNeely MJ, Fujimoto WY, Kahn SE: Oral disposition index predicts the development of future diabetes above and beyond fasting and 2-h glucose levels. Diabetes Care 2009, 32(2):335-341.

http://www.doi.org/10.2337/dc08-1478

68. Neese JW, Duncan P, Bayse D, Robinson M, Cooper T, Stewart C: Development and evaluation of a hexokinase glucose-6-phosphate dehydrogenase procedure for use as a national glucose reference method (Publication No (CDC) 77-8330). US Department of Health Education and Welfare 1976.

69. El Kenz H, Bergmann P: Evaluation of immunochemiluminometric assays for the measurement of insulin and C-peptide using the ADVIA Centaur ${ }^{\circledast}$. Clinical Laboratory 2004, 50(3-4):171-174.

70. Moore MC, Davis SN, Mann SL, Cherrington AD: Acute fructose administration improves oral glucose tolerance in adults with type 2 diabetes. Diabetes Care 2001, 24(11):1882-1887.

http://www.doi.org/10.2337/diacare.24.11.1882

71. Moore MC, Cherrington AD, Mann SL, Davis SN: Acute fructose administration decreases the glycemic response to an oral glucose tolerance test in normal adults. Journal of Clinical Endocrinology and Metabolism 2000, 85(12):4515-4519.

http://www.doi.org/10.1210/jc.85.12.4515
72. Buchanan TA, Xiang AH, Peters RK, Kjos SL, Marroquin A, Goico J, Ochoa C, Tan S, Berkowitz K, Hodis HN, Azen SP: Preservation of pancreatic beta-cell function and prevention of type 2 diabetes by pharmacological treatment of insulin resistance in high-risk Hispanic women. Diabetes 2002, 51(9):2796-2803.

http://www.doi.org/10.2337/diabetes.51.9.2796

73. Kahn SE: The relative contributions of insulin resistance and beta-cell dysfunction to the pathophysiology of type 2 diabetes. Diabetologia 2003, 46(1):3-19. http://www.doi.org/10.1007/s00125-002-1009-0

74. Buchanan TA: Pancreatic beta-cell loss and preservation in type 2 diabetes. Clinical Therapeutics 2003, 25(Suppl B):B32-B46.

http://www.doi.org/10.1016/s0149-2918(03)80241-2

75. Salunkhe VA, Veluthakal R, Kahn SE, Thurmond DC: Novel approaches to restore beta cell function in prediabetes and type 2 diabetes. Diabetologia 2018, 61(9):1895-1901. http://www.doi.org/10.1007/s00125-018-4658-3

76. Gonzalez M, Zarzuelo A, Gamez MJ, Utrilla MP, Jimenez J, Osuna I: Hypoglycemic activity of olive leaf. Planta Medica 1992, 58(6):513-515. http://www.doi.org/10.1055/s-2006-961538

77. Cumaoglu A, Rackova L, Stefek M, Kartal M, Maechler P, Karasu C: Effects of olive leaf polyphenols against $\mathrm{H} 2 \mathrm{O} 2$ toxicity in insulin secreting beta-cells. Acta Biochimica Polonica 2011, 58(1):45-50.

http://www.doi.org/10.18388/abp.2011_2284

78. Koch ER, Deo P: Nutritional supplements modulate fluorescent protein-bound advanced glycation endproducts and digestive enzymes related to type 2 diabetes mellitus. Bmc Complementary and Alternative Medicine 2016, 16(1):1-7.

http://www.doi.org/10.1186/s12906-016-1329-0

79. Yang XP, Kong FB: Effects of tea polyphenols and different teas on pancreatic alpha-amylase activity in vitro. LwtFood Science and Technology 2016, 66:232-238. http://www.doi.org/10.1016/j.Iwt.2015.10.035

80. Zhou PY, Zhang LM, Li W, Zhang ST, Luo LX, Wang J, Sun BS: In vitro evaluation of the anti-digestion and antioxidant effects of grape seed procyanidins according to their degrees of polymerization. Journal of Functional Foods 2018, 49:85-95.

http://www.doi.org/10.1016/j.jff.2018.08.001

81. Ryan CM, Khoo W, Stewart AC, O'Keefe SF, Lambert JD, Neilson AP: Flavanol concentrations do not predict 
dipeptidyl peptidase-IV inhibitory activities of four cocoas

with different processing histories. Food and Function

2017, 8(2):746-756.

http://www.doi.org/10.1039/c6fo01730d

82. Bartoli E, Fra GP, Schianca GPC: The oral glucose tolerance test (OGTT) revisited. European Journal of Internal Medicine 2011, 22(1):8-12.

http://www.doi.org/10.1016/j.ejim.2010.07.008

83. Abdul-Ghani MA, Jenkinson CP, Richardson DK, Tripathy $D$, DeFronzo RA: Insulin secretion and action in subjects with impaired fasting glucose and impaired glucose tolerance Results from the veterans administration genetic epidemiology study. Diabetes 2006, 55(5):1430-1435.

http://www.doi.org/10.2337/db05-1200

84. Hanefeld M, Koehler C, Fuecker K, Henkel E, Schaper F, Temelkova-Kurktschiev T: Insulin secretion and insulin sensitivity pattern is different in isolated impaired glucose tolerance and impaired fasting glucose - The risk factor in impaired glucose tolerance for atherosclerosis and diabetes study. Diabetes Care 2003, 26(3):868-874. http://www.doi.org/10.2337/diacare.26.3.868

85. Marini MA, Frontoni S, Succurro E, Arturi F, Sciacqua A, Hribal ML, Perticone F, Sesti G: Insulin sensitivity, and betacell function in relation to hemoglobin A1C. Nutrition
Metabolism and Cardiovascular Diseases 2014, 24(1):27-

33.

http://www.doi.org/10.1016/j.numecd.2013.01.011

86. Edelman D, Olsen MK, Dudley TK, Harris AC, Oddone EZ: Utility of hemoglobin A1c in predicting diabetes risk. Journal of General Internal Medicine 2004, 19(12):11751180.

http://www.doi.org/10.1111/j.1525-1497.2004.40178.x

87. Loopstra-Masters RC, Haffner SM, Lorenzo C, Wagenknecht LE, Hanley AJ: Proinsulin-to-C-peptide ratio versus proinsulin-to-insulin ratio in the prediction of incident diabetes: the Insulin Resistance Atherosclerosis Study (IRAS). Diabetologia 2011, 54(12):3047-3054. http://www.doi.org/10.1007/s00125-011-2322-2

88. Jones CN, Pei D, Staris P, Polonsky KS, Chen YD, Reaven GM: Alterations in the glucose-stimulated insulin secretory dose-response curve and in insulin clearance in nondiabetic insulin-resistant individuals. Journal of Clinical Endocrinology and Metabolism 1997, 82(6):1834-1838. http://www.doi.org/10.1210/jc.82.6.1834

89. Jones AG, Hattersley AT: The clinical utility of C-peptide measurement in the care of patients with diabetes. Diabetic Medicine 2013, 30(7):803-817. http://www.doi.org/10.1111/dme.12159 